\title{
Benigne Prostatahyperplasie: enukleieren statt vaporisieren
}

_ Eine deutlich kürzere Behandlungsdauer und die Reduktion der Behandlungskosten - das verspricht das neue Therapieverfahren mit dem GreenLight ${ }^{\mathrm{TM}}$-Laser bei mittleren bis großen Prostata-Adenomen. Bei der „GreenLight ${ }^{T M}$ Enucleation of the Prostate“ (GreenLEP) werden die Gewebestücke nicht vaporisiert, sondern blutungsarm enukleiert und anschließend morcelliert. Der spanische Urologe Dr. Fernando Gómez Sancha hat das Verfahren in Zusammenarbeit mit dem deutschen Medizintechnikhersteller Richard Wolf entwickelt. Sancha erläutert: „Die Vaporisation der Prostata mittels des Green Light ${ }^{\mathrm{TM}}$-Lasers ist inzwischen zur Standard-Methode in vielen Kliniken geworden. Mir fiel auf, dass die Behandlung von großen Adenomen, obwohl mittels Vaporisation technisch möglich, zu viele Fasern verbrauchte, wodurch sich die Behandlungszeiten deutlich verlängerten und die Kosten erhöhten, und trotzdem die Ergebnisse alternativer Therapien wie der Behandlung mit dem HoLEP-
Laser schwer zu erreichen waren. Die Enukleation mittels des GreenLight ${ }^{\mathrm{TM}}$-Lasers erlaubt nun die komplette Behandlung des adenomatösen Gewebes.“

Für das neue Therapieverfahren bietet die Firma Richard Wolf ein optimiertes Instrumenten- und Geräte-Set an. Zum einen das „Freestyle“-Laser-Instrument, das von den Anwendern vor allem wegen der Bewegungsfreiheit sowie der sehr guten Sicht aufgrund eines optimalen Spülverhältnisses und einer patentierten, vibrationsfreien Faserführung geschätzt wird. Zum anderen den leistungsstarken und sicheren „Piranha“-Gewebemorcellator, der die enukleierten Gewebestücke in der Blase ansaugt, zerkleinert und anschließend absaugt. Dabei bleibt das morcellierte Gewebe, welches in einem Auffangbehälter gesammelt wird, groß genug, um es anschließend histologisch untersuchen zu können.

Nach Informationen von

Richard Wolf, Knittlingen

\section{Denosumab in der Europäischen Union zugelassen}

— Seit Ende Mai ist der vollhumane monoklonale Antikörper Prolia ${ }^{\circledR}$ (Denosumab) von der Europäischen Kommission in zwei Indikationen zugelassen. Erstens zur Behandlung der Osteoporose bei postmenopausalen Frauen mit erhöhtem Frakturrisiko. Zweitens zur Behandlung eines Knochendichteverlustes durch eine hormonablative Therapie (Androgendeprivation) bei Männern mit Prostatakarzinom und erhöhtem Frakturrisiko. Für die zweite Indikation ist Denosumab das erste und einzige zugelassene Produkt.

Die Marktzulassung stützt sich auf die Ergebnisse von sechs Phase-III-Studien. Diese Untersuchungen zeigen, dass Denosumab die Knochendichte im gesamten Skelett erhöht. In zwei zulassungsrelevanten Studien bei postmenopausalen Frauen mit Osteoporose sowie bei Männern mit Prostatakarzinom und Knochenverlust unter Androgendeprivationstherapie waren außerdem neue
Frakturen als Endpunkte festgelegt worden. Die Ergebnisse belegen, dass eine zweimal jährliche subkutane Injektion von $60 \mathrm{mg}$ Denosumab die Inzidenz von Knochenbrüchen reduziert. Alle sechs Studien zeigten, dass Denosumab die Knochendichte an allen untersuchten Skelettlokalisationen erhöht. In der HALT-Studie (Hormone Ablation Bone Loss Trial) wurde untersucht, wie sich die Knochendichte der Lendenwirbelsäule unter Denosumab beziehungsweise unter Placebo im Vergleich zum Ausgangswert veränderte. 1.468 Männer, die aufgrund eines nicht metastasierten Prostatakarzinoms eine Androgendeprivationstherapie erhielten, nahmen an der Studie teil. Nach 36 Monaten hatten Patienten, die mit Denosumab anstatt mit Placebo behandelt wurden, ein um $62 \%$ verringertes Risiko für neue Wirbelkörperfrakturen.

Nach Informationen von Amgen, München

\section{Neues Propiverin-Präparat}

Seit 1. September hat die mibe $\mathrm{GmbH}$ ihr Präparateprogramm um das Spasmolytikum Propiver ${ }^{\circledR}$ (Propiverin) erweitert. Propiver ${ }^{\circledR}$ ist als 5-mg- und als 15 -mg- Filmtablette in den Packungsgrößen 28 (N1), 49 (N2) und $98\left(\mathrm{~N}_{3}\right)$ Stück erhältlich. Das neue Medikament verfügt über eine hohe Rabattvertragsabdeckung. Als zusätzlichen Service können Interessierte Miktionstagebücher kostenlos anfordern.

Nach Informationen von mibe, Jena

Therapieoption bei chronischer Zystitis zum Einführungspreis

Mit Uropol ${ }^{\circledR}$ (400 mg Natrium-Chondroitinsulfat) steht $a b$ sofort eine neue Therapieoption zur Regeneration der GAG-Schicht zur Verfügung, die bei chronischer Zystitis oder überaktiver Blase geschädigt sein kein. Bei einer Instillationsmenge von $20 \mathrm{ml}$ hat Uropol ${ }^{\circledR}$ die höchste derzeit am Markt verfügbare Dosierung. Vier Uropo $^{\circledR}$-Vials à $20 \mathrm{ml}$ sind bis Ende Dezember zum Einführungspreis von 199,60 EUR (statt 349,96 EUR) erhältlich.

Nach Informationen von Medac, Wedel

\section{Neues Mitomycin-Präparat auf dem Markt}

Seit 1. August steht Urocin ${ }^{\circledR}(20 \mathrm{mg}$ Mitomycin) von Apogepha für die intravesikale Instillationstherapie des nicht invasiven Harnblasenkarzinoms zur Verfügung. Das mit $\mathrm{NaCl}$ befüllte Kompakt-System inklusive Katheter und Entsorgungsbeutel eignet sich für die individuelle Therapie und erfüllt die Anforderungen der Berufsgenossenschaft an ein geschlossenes System. Eine Glasflaschenummantelung (Kontaminations- und Splitterschutz) bietet zusätzliche Sicherheit. Urocin $^{\circledR}$ wird in drei Packungsgrößen angeboten (eine, vier oder sechs Durchstechflaschen) und bietet in der größten einen Zuzahlungsvorteil für GKV-Patienten.

Nach Informationen von Apogepha, Dresden 